\title{
Toxoplasma gondii Inside LLCMK2 Cells. A Study by Field Emission Scanning and Transmission Electron Microscopy
}

\author{
Rodrigo Cardoso Magno, Lorian Cobra Straker, Wanderley de Souza, and Marcia Attias \\ Instituto de Biofísica Carlos Chagas Filho, UFRJ. 21949-900. Rio de Janeiro, RJ Brazil \\ mattias@biof.ufri.br
}

INTRODUCTION: Transmission electron microscopy (TEM) of ultrathin sections has been, for decades, the classical tool for studying intracellular structures and organelles. The intrinsic limitations of bi-dimensional images have been partially overcome by 3 -D reconstruction from serial sections. The onset of field emission scanning electron microscopy (FESEM) enhanced the resolution power of SEM to a level that allowed the observation of fine cellular details that could not be discriminated before. Aiming the exposure of inner structures and thin conductive coating of the sample, new technical approaches were developed for specimen preparation for FESEM. Cleavage of frozen specimens, followed by maceration in $\mathrm{OsO}_{4}$ as proposed by [1], and others required the use of compact tissue. For cell monolayers, rupturing methods before and after drying were proposed by several authors $[2,3,4]$. In this study, monolayers of LLCMK2 cells at various stages of infection by the protozoan parasite Toxoplasma gondii were observed by TEM of random or serial thin sections from flat embedded samples and by FESEM after dry scraping and removal of the upper plasma membrane with scotch tape.

Toxoplasma gondii is an obligatory intracellular parasite of warm blooded animals, agent of human toxoplasmosis [5]. This parasite can invade and multiplicate assexually in any nucleated cell. Gametogenesis and sexual mating occurs only in gut epithelial cells of felines. LLCMK2 is a cell lineage derived from kidney epithelial cells of Macaca mulatta. LLCMK2 was selected for experiments because it is not a natural phagocyte, so active invasion would be the only possibility of cell invasion by $T$. gondii.

M\&M: 50:1 parasites were added and challenged for 15 minutes over LLCMK2 monolayers. After rinsing with culture medium (RPMI) to remove parasites from the extracellular medium, cultures were incubated for 1,7 and 24 hours post-infection and fixed overnight at $4^{\circ} \mathrm{C}$ in $2.5 \%$ glutaraldehyde in $0.1 \mathrm{M}$ cacodylate buffer $\mathrm{pH} 7.2$; containing $3 \%$ sucrose. Post-fixation was done in $1 \% \mathrm{OsO}_{4}$ in the same buffer. For FESEM, after $\mathrm{CO}_{2}$ critical point drying, coverslips with adhered cells were gently scraped with Scotch tape, exposing the cytoplasm and the parasitophorous vacuole, where the parasites multiplicate. TEM observations were made on a ZEISS 900EM, and FESEM observations in a Jeol JSM6340.

RESULTS AND DISCUSSION: 1 hour after challenge (fig. 1) each LLCMK2 cell contained one or more parasitophorous vacuoles (PV), each containing a single parasite (tx). After 7 hours they had already finished the first endodiogenic division and 24 hours after challenge (fig. 2) each PV contained a large number of parasites (20 or more). At the FESEM we observed that in each vacuole all parasites remained attached by the posterior end to a residual body (rb), forming a rosette (figs. 2 and 4). The nanotubules (nt) that form the intravacuolar network (IVN) connected the parasites to each other, apparently helping to keep the rosette. Connections of the IVN with the membrane of the PV were also seen. Besides the IVN tubules, other membranes $\left(^{*}\right)$ were observed inside the PV(fig. 3). These seem to be related to the PV membrane and profiles of the endoplasmic reticulum that surround it. Information obtained from FESEM images correlates well with thin sections from monolayers. These data suggest to us that: 1- the IVN contributes in maintaining the parasites attached to the residual body until early before the host cell ruptures, 2- 
The PVs of a single cell do not fuse to each other, 3- Membranes derived from the ER and PVM are also internalized in the PV; 4- The observation of fractured monolayers in FESEM is a valuable tool to the study of the intracellular development of $T$. gondii.

Supports: CNPq-PRONEX, Faperj, CAPES REFERENCES

[1] Tanaka K, Mitsuhima A J. Microsc. 133 (1984)

[2] Peters, KR, Carley, WM. Scan Microsc(2) (1988)

[3] Haggis, GH. Scan Electron Microsc (suppl. 3) (1989)

[4] Flood,PR. Scan Electron Microsc I/IITRI (1975)

[5]Dubey et al., Clin. Microbiol. Rev. 11(2): 267-299. 1998

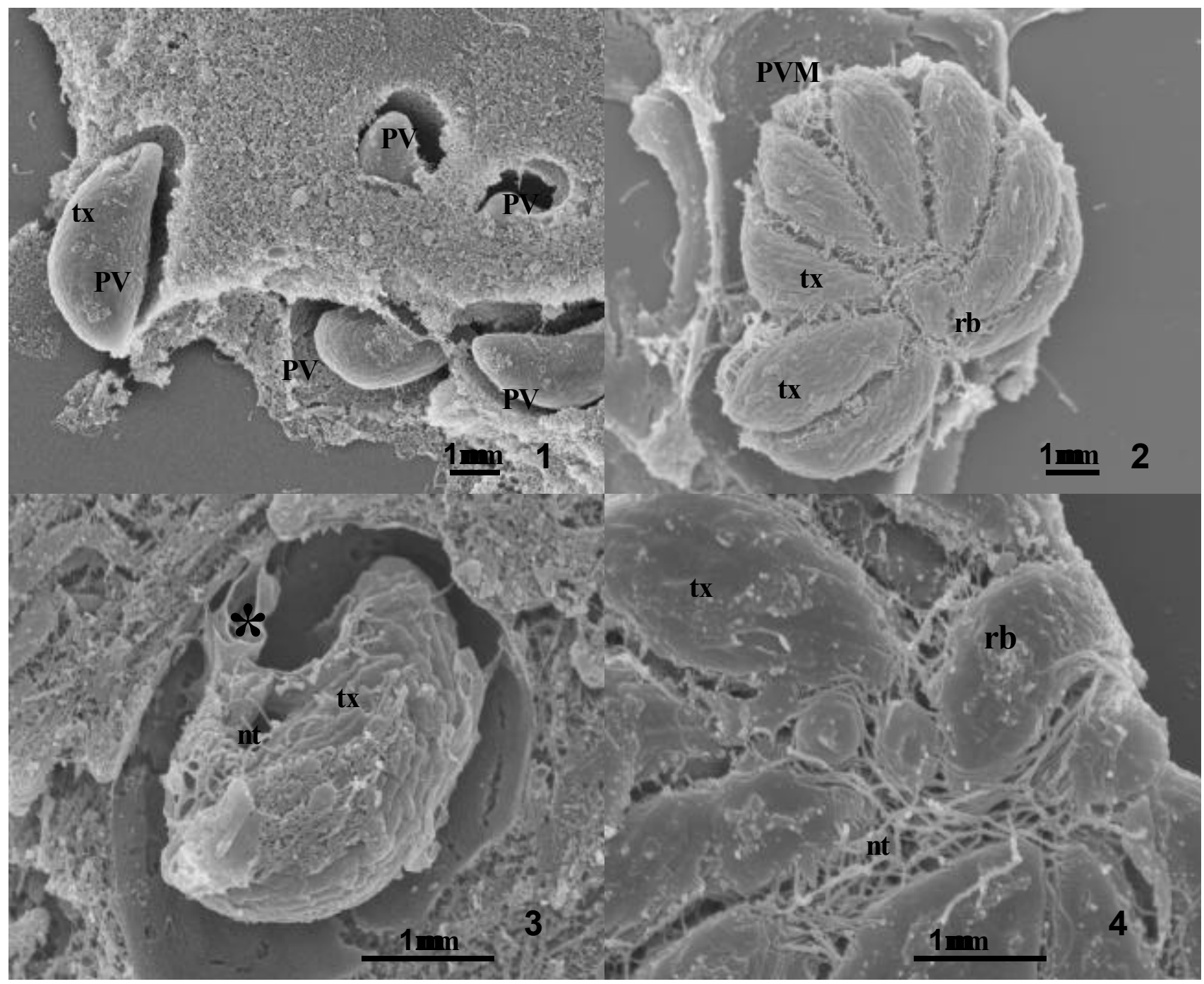

